\title{
Biological evaluation of intervertebral disc cells in different formulations of gellan gum-based hydrogels
}

\author{
G Khang ${ }^{1,2 *}$, SK Lee ${ }^{1}$, HN Kim² ${ }^{2}$ J Silva-Correia ${ }^{3,4}$, ME Gomes ${ }^{3,4}$, CAA Viegas ${ }^{3,4,5}$, IR Dias ${ }^{3,4,5}$, \\ JM Oliveira ${ }^{3,4}$ and RL Reis ${ }^{3,4}$ \\ ${ }^{1}$ Chonbuk National University, Department of Polymer-Nano Science and Technology, Jeonju, Korea \\ ${ }^{2}$ Chonbuk National University, Department of BIN Fusion Technology, Jeonju, Korea \\ ${ }^{3} 3$ B's Research Group - Biomaterials, Biodegradables and Biomimetics, Univ. Minho, Headquarters of the European Institute of Excellence \\ on Tissue Engineering and Regenerative Medicine, AvePark, S. Cláudio de Barco, 4806-909, Taipas, Guimarães, Portugal \\ ${ }^{4}$ ICVS/3B's - PT Government Associate Laboratory, Braga/Guimarães, Portugal \\ ${ }^{5}$ Department of Veterinary Sciences, University of Trás-os-montes and Alto Douro, Vila Real, Portugal
}

\begin{abstract}
Gellan gum (GG)-based hydrogels are advantageous in tissue engineering not only due to their ability to retain large quantities of water and provide a similar environment to that of natural extracellular matrix (ECM), but also because they can gelify in situ in seconds. Their mechanical properties can be fine-tuned to mimic natural tissues such as the nucleus pulposus (NP). This study produced different formulations of GG hydrogels by mixing varying amounts of methacrylated (GG-MA) and high-acyl gellan gums (HA-GG) for applications as acellular and cellular NP substitutes. The hydrogels were physicochemically characterized by dynamic mechanical analysis. Degradation and swelling abilities were assessed by soaking in a phosphate buffered saline solution for up to $170 \mathrm{~h}$. Results showed that as HA-GG content increased, the modulus of the hydrogels decreased. Moreover, increases in HA-GG content induced greater weight loss in the GG-MA/HA-GG formulation compared to GG-MA hydrogel. Potential cytotoxicity of the hydrogel was assessed by culturing rabbit NP cells up to 7 days. An MTS assay was performed by seeding rabbit NP cells onto the surface of 3D hydrogel disc formulations. Viability of rabbit NP cells encapsulated within the different hydrogel formulations was also evaluated by Calcein-AM and ATP assays. Results showed that tunable GG-MA/HA-GG hydrogels were non-cytotoxic and supported viability of rabbit NP cells. Copyright (C) 2012 John Wiley \& Sons, Ltd.
\end{abstract}

Received 27 August 2012; Accepted 28 August 2012

Keywords gellan gum; nucleus pulposus; glycidyl methacrylate; hydrogel; high-acyl gellan gum (HA-GG)

\section{Introduction}

Tissue engineering is an approach that enables the formation of new parenchyma by implanting a polymer scaffold, which is then completely absorbed in vivo to result in tissue affinity (Khang G et al., 2002). The role of polymer scaffolds in tissue engineering applications is to generate differentiated tissue by activating cells and promoting secretion of new extracellular matrix (ECM) (Khang G

\footnotetext{
*Correspondence to: G. Khang, Chonbuk National University, Department of Polymer-Nano Science and Technology, Jeonju, Korea.E-mail: gskhang@chonbuk.ac.kr
}

et al., 2006). In addition, the polymer is usually composed of biodegradable materials that enable attachment, proliferation and viability of cells (Hu SG et al., 2003; Murphy WL et al., 2000). This biomaterial can also function as a delivery agent for biological factors, thereby facilitating the accumulation of tissues and cells and imitating ECM in the body (Weiner AA et al., 2008).

Low back pain is the second leading cause of disability in 30-50 year-olds, although global prevalence has been shown to be more in the 60-80 year range (Fritz JM et al., 2008). Intervertebral disc (IVD) degeneration is associated with healthcare expenditures of $>90$ billion dollars annually in the USA (Manchikanti L et al., 2009). The histological structure of an IVD is comprised of an 
annulus fibrous (AF) and a nucleus pulposus (NP), both of which are structurally limited by terminal cartilage joints (cartilaginous endplates, CEP). The IVD is supported by the ECM of AF and NP. AF is the outer histological compartment of IVD, which is mainly composed of collagen type I. The structural arrangement of the collagen fibres in concentric lamellae around the NP provides compressive strength (Bibby SRS et al., 2001). The NP is located at the centre of the IVD and consists of a tissue matrix containing large amounts of collagen type II and a high proportion of proteoglycans that provide a gel-like hydrophilicity to the structure (Bogduk N, 1997). Tissue engineering for regeneration of IVD requires the use of different scaffolds. For regeneration of the NP, a scaffold must be able to withstand loads and allow easy and minimally invasive implantation of cellular and cell-loaded scaffolds (Lee and Mooney, 2001).

Hydrogels have interesting properties for IVD regeneration such as the ability to maintain high water content and to be inserted and formed in situ through a minimally invasive procedure (Chung and Park, 2009; Hoffman AS, 2002; Pereira et al., 2011a). Gellan gum (GG)-based hydrogels have received particular attention in the field of cartilage regeneration (Gong YH et al., 2009; Oliveira JT et al., 2009a; Oliveira JT et al., 2009b; Oliveira JT et al., 2010) and more recently in intervertebral disc regeneration (Silva-Correia et al., 2010; Silva-Correia et al., 2011; Pereira et al., 2011a). GG, one of the few natural polymers approved by the FDA as a food additive, is a microbial polysaccharide produced by Pseudomonas elodea. This natural polymer consists of repeating units of tetrasaccharide; $1,3-\beta-\mathrm{D}$-glucose; 1,4- $\beta$-D-glucuronic acid; $1,4-\beta$-D-glucose; and 1,4- $\alpha$-D-Lrhamnose (Jansson PE et al., 1983; O’Neill MA et al., 1983). GG is soluble in water at high temperatures $\left(\sim 90^{\circ} \mathrm{C}\right)$ and forms a transparent gel at $\sim 30-35^{\circ} \mathrm{C}$ (Nakamura $\mathrm{K}$ et al., 1993). To form a stable hydrogel, cations must be present in the solution for sol-gel transition (Matricardi P et al., 2009). GG is available in two different forms of acylation: 1) a high-acyl form with two acyl substituents, both located on the same glucose residue (one glycerate per repeat and one acetate per every two repeats) that produces soft, elastic and non-brittle gels; and 2) a low-acyl form with no acyl groups that produces firm, non-elastic brittle gels. GG can provide abundant gel texture using very small quantities of material (Sanderson GR, 1990), thereby making it one of the most promising in situ gelling materials for human body applications and biomedical technologies (Coviello $\mathrm{T}$ et al., 1998; Nanjawade BK et al., 2007).

GG hydrogels have been shown to support the growth and ECM deposition of human articular chondrocytes in vitro and in vivo (Oliveira JT et al., 2009a; Oliveira JT et al., 2009b; Oliveira JT et al., 2010). The main problems with tissue-engineered applications are their weak mechanical properties and uncontrolled degradation rates (Gong YH et al., 2009). As a result, promising substitutes based on chemically modified GG have been recently proposed for NP regeneration (Silva-Correia $\mathrm{J}$ et al., 2010; Silva-Correia J et al., 2011). Stable and non-cytotoxic ionic and photo crosslinked methacrylated GG hydrogels were developed with improved mechanical properties and better temporal/spatial control over polymerization, which occurs by immersion in a saline solution at physiological $\mathrm{pH}$ and/or by exposure to UV light. In another approach, a new structure using microparticles/matrices composed of different formulations of low- and high-acyl GG was developed as an NP substitute (Pereira et al., 2011b). The structure enabled better control of mechanical properties by combining the two forms of GG at different ratios and by cellular encapsulation and dispersion within the system.

These recent studies have led to improvements in GG properties with the aim of using these new biomaterials for applications in tissue engineering of NP. In the current study, we hypothesized a combination of both approaches: 1) the use of formulations that combined two types of GG with different stabilities and permeabilities and 2) the use of a chemically modified form of GG. As a result, ioniccrosslinked hydrogel discs with different in vitro stabilities were produced by combining $5 \mathrm{~g}$ of chemically modified materials such as high-acyl gellan gum (HA-GG) and methacrylated low-acyl gellan gum (GG-MA). Hydrogel discs were physico-chemically characterized in terms of mechanical properties and degradation/swelling ability. In addition, screening of the biocompatibilities of these GG-based formulations was assessed in vitro using rabbit cells isolated from IVD, namely NP cells, as well as by observing interactions with cells. In fact, we evaluated in vitro the performance of formulations in two stages: 1) by culture of NP cells in the presence of GG-based hydrogel leachables and 2) by encapsulating NP cells within the hydrogels.

\section{Materials and methods}

\subsection{Preparation of GG-based hydrogel formulations}

Different GG-based formulations were produced by combining high-acyl gellan gum (HA-GG) and methacrylated low-acyl gellan gum (GG-MA) at different percentages. A solution of HA-GG composed of $\mathrm{KelcoGel}^{\circledR}$ LT100 with $M_{w}=1-2 \times 10^{6}$ Da (CP Kelco, Atlanta, GA, USA) was prepared in distilled water at a $0.75 \%(\mathrm{w} / \mathrm{v})$ final concentration. A homogenous solution was obtained after constant stirring for $24 \mathrm{~h}$ at room temperature. GG-MA powder $\left(\mathrm{M}_{\mathrm{W}}=62.4 \mathrm{kDa}\right)$ was synthesized as described by SilvaCorreia et al. (2011) and dissolved in distilled water with vigorous stirring to yield a final concentration of $2 \%(\mathrm{w} / \mathrm{v})$. Different formulations of hydrogels were obtained by combining $0.75 \%(\mathrm{w} / \mathrm{v})$ HA-GG and $2 \%(\mathrm{w} / \mathrm{v})$ GG-MA aqueous solutions at ratios of $75: 25 \%, 50: 50 \%$ and 25\%:75\% (v/v) (HA-GG : GG-MA). Preparations were performed at room temperature under constant stirring for 24 h. The formulations were then used to produce hydrogel discs with appropriate dimensions according to the final test.

In addition, a low-acyl gellan gum (LA-GG) solution was prepared according to a procedure described 
by Silva-Correia et al. (2011). Briefly, LA-GG powder composed of Gelzan ${ }^{\mathrm{TM}}$, with $\mathrm{M}_{\mathrm{w}}=2-3 \times 10^{5}$ Da (Sigma-Aldrich Co., St-Louis, MI, USA) was dissolved in distilled water to obtain a final concentration of $2 \%$ (w/v). The mixture was heated to $90^{\circ} \mathrm{C}$ with continuous stirring and complete dissolution was achieved after $30 \mathrm{~min}$. Next, temperature was decreased to $60-65^{\circ} \mathrm{C}$ and PBS pH 7.4 (Phosphate Buffered Saline, SigmaAldrich) solution was added to a final concentration of $10 \%$ (v/v) in LA-GG solution. Following a slow decrease in temperature to $50^{\circ} \mathrm{C}$ under constant stirring, LA-GG discs were prepared by transferring the solution to a silicone mould and allowing gelation to occur at room temperature for $\sim 5 \mathrm{~min}$. All discs examined were subsequently stabilized in PBS for a minimum of $30 \mathrm{~min}$.

\subsection{Characterization of GG-based hydrogel formulations}

\subsubsection{Dynamic mechanical analysis (DMA)}

Dynamic mechanical analysis (DMA) was performed to characterize the mechanical behaviour of the different formulations of hydrogel discs by incorporating HA-GG and ioniccrosslinked GG-MA. The formulations were obtained by combining $0.75 \%(\mathrm{w} / \mathrm{v})$ HA-GG and $2 \%(\mathrm{w} / \mathrm{v})$ GG-MA aqueous solutions at ratios of 50:50\% and 25:75\% (v/v). Discs made of $2 \%$ (w/v) GG-MA were used as controls. Viscoelastic measurements were obtained at $37^{\circ} \mathrm{C}$ using a TRITEC8000B DMA instrument from Triton Technology Ltd. (Lincolnshire, UK) equipped with a compressive mode. Samples were produced using cylindrical silicone moulds of 7-mm diameter and 4-mm height measured for each sample. Hydrogel discs were immersed in PBS ( $\mathrm{pH}$ 7.4) until assayed and then analyzed while immersed in a liquid bath in a Teflon ${ }^{\circledR}$ reservoir. Geometry of the samples was measured and samples were clamped and immersed in PBS (pH7.4). DMA spectra were obtained by frequency scans of 0.1 to $10 \mathrm{~Hz}$ following equilibration at $37^{\circ} \mathrm{C}$. Measurements were performed under a constant $50 \mu \mathrm{m}$ amplitude. A small preload was applied to each sample to ensure that the entire disc surface was in contact with the compression plates before testing and that the distance between plates was equal for all scaffolds tested. Three samples were used for each condition $(n=3)$.

\subsubsection{Degradation and swelling ability}

Hydrogel discs from the formulations were prepared according to the procedure described above in a 48-well tissue-cultured polystyrene (TCPS) plate using $125 \mathrm{~mL}$ per disc and lyophilized afterwards. Initial dry weight $\left(\mathrm{W}_{\mathrm{di}}\right)$ of the samples was then determined and the discs were incubated in $2 \mathrm{~mL}$ PBS (pH 7.4) at $37^{\circ} \mathrm{C}$ under mild shaking. Wet weight $\left(\mathrm{W}_{\mathrm{w}}\right)$ was measured after incubation at different time points. Next, samples were freeze-dried again and a final dry weight $\left(\mathrm{W}_{\mathrm{df}}\right)$ was determined. Degradation $\left(\mathrm{D}_{\mathrm{r}}\right)$ and swelling $\left(\mathrm{S}_{\mathrm{r}}\right)$ ratios were calculated according to Equations (1) and (2), respectively:

$$
\begin{aligned}
& \mathrm{D}_{\mathrm{r}}(\%)=100 \mathrm{x}\left(\mathrm{W}_{\mathrm{di}}-\mathrm{W}_{\mathrm{df}}\right) / \mathrm{W}_{\mathrm{di}} \\
& \mathrm{S}_{\mathrm{r}}(\%)=100 \mathrm{x}\left(\mathrm{W}_{\mathrm{w}}-\mathrm{W}_{\mathrm{di}}\right) / \mathrm{W}_{\mathrm{di}}
\end{aligned}
$$

\subsection{Biological evaluation of GG-based hydrogel formulations}

\subsubsection{Isolation NP cells from rabbit IVD}

Hydrogel formulations were biologically evaluated using NP cells isolated from New Zealand White Rabbit IVDs. The New Zealand White Rabbit is the result of Albino rabbit outbreeding and is a mid-sized animal often used in animal experiments, particularly in IVD regeneration studies. NP cells were isolated from a 4-week-old female animal using an enzymatic-based method (collagenase type A). Briefly, after animal sacrifice, extracted discs were placed in PBS (pH 7.4) and washed several times with PBS containing a $1 \%(\mathrm{v} / \mathrm{v})$ antibiotic-antimycotic mixture until complete removal of blood and other bodily contaminants. NP tissue was then collected from the middle portion of the IVD, separated from any obvious dense annulus tissue and immersed in PBS. After washing several times in PBS, NP tissue digestion was performed by incubation at $37^{\circ} \mathrm{C}$ in a humidified atmosphere of $5 \% \mathrm{CO}_{2}$ for $24 \mathrm{~h}$ in $10 \mathrm{~mL} \mathrm{Gibco}{ }^{\circledR}$ Dulbecco's modified Eagle's Medium (DMEM F12, Life Technologies $^{\mathrm{TM}}$ Inc., Invitrogen, Burlington, ON, Canada) containing 0.3\% (v/v) collagenase type A (Roche Applied Science, Mannheim, Germany) and supplemented with $10 \%$ (v/v) fetal bovine serum (FBS) (Biochrom AG, Berlin, Germany), $1 \%$ (v/v) of an antibiotic-antimycotic mixture (Invitrogen) containing 10000 units/mL Penicillin G Sodium, $10000 \mathrm{mg} / \mathrm{ml}$ Streptomycin sulphate and $25 \mathrm{mg} / \mathrm{ml}$ amphotericin B (Fungizone ${ }^{\circledR}$ Antimycotic 0.85\% saline; Invitrogen). A 100-mm cell strainer was used to separate the cells from the remaining tissue debris. Finally, the isolated NP cells were expanded in the DMEM:F12 (1:1) medium supplemented with $10 \%(\mathrm{v} / \mathrm{v})$ FBS and $1 \%(\mathrm{v} / \mathrm{v})$ of the antibiotic-antimycotic mixture under standard culture conditions until confluence. The medium was changed every three days.

\subsubsection{In vitro cytotoxicity screening}

Investigation of the potential effect of different formulations of GG-based hydrogels on NP cellular metabolism was performed using a standard 3-(4,5-dimethylthiazol-2-yl)5(3-carboxymethoxyphenyl)-2(4-sulfofenyl)-2H-tetrazolium (MTS) viability test in accordance with ISO/EN 10993 (2009) Part 5 guidelines. All materials were processed under sterile conditions using powders sterilized by ethylene oxide to produce discs in 48-well TCPS plates with $125 \mathrm{~mL}$ per disc. The MTS viability test was performed after contact of NP cells with the leachables, which were released from the materials within $24 \mathrm{~h}$ of extraction and following seeding of NP cells on top of the hydrogel discs.

MTS assay in 2D cultures. Extraction fluids were prepared by introducing a minimum of 41 discs with diameters of $15 \mathrm{~mm}$ 
in $50 \mathrm{~mL}$ flasks containing $20 \mathrm{~mL}$ DMEM:F12 (1:1) medium in a thermostatic bath at $37^{\circ} \mathrm{C}$ and spun at $60 \mathrm{rpm}$ for $24 \mathrm{~h}$. NP cells were grown as monolayers as described above. After two passages, confluent cells were detached from the flasks using trypsin (0.25\% trypsin/EDTA solution, Sigma-Aldrich) and a diluted suspension was centrifuged at $1200 \mathrm{rpm}$ for 5 min. NP cells were seeded in each well of a 96-well TCPS plate (six replicates per sample) at a density of $2 \times 10^{4}$ cells/ well and cultured for $24 \mathrm{~h}$ at $37^{\circ} \mathrm{C}$ in a $5 \% \mathrm{CO}_{2}$ atmosphere. Next, cells were incubated with $200 \mathrm{~mL}$ of each extract after reaching $80-90 \%$ confluence. The MTS test was performed after 1,3 and 7 days of culture at $37^{\circ} \mathrm{C}$ and $5 \% \mathrm{CO}_{2}$ by removing the extracts and adding $300 \mathrm{~mL}$ to each well of a mixture containing serum-free culture medium without Phenol Red and MTS (CellTiter ${ }^{\circledR} 96$ AQueous One Solution Cell Proliferation Assay kit; Promega, Madison, WI, USA) at a ratio of 5:1. Positive and negative controls such as latex rubber extract and culture medium were also used. Optical density (OD) was measured at $490 \mathrm{~nm}$ using a plate reader (Molecular Devices, Sunnyvale, CA, USA) after incubation for $3 \mathrm{~h}$ at $37^{\circ} \mathrm{C}$ and $5 \% \mathrm{CO}_{2}$ atmosphere. The percentage of cell viability was calculated by normalization with the mean OD value obtained for the negative control. All tests were performed in triplicate $(n=18)$.

MTS assay on 3D scaffolds. Confluent NP cells ( $5 \times 10^{4}$ cells/ disc) were seeded in the top $15-\mathrm{mm}$ diameter hydrogel discs. The constructs were then incubated with $1 \mathrm{~mL}$ DMEM:F12 (1:1) medium for 1,3 and 7 days at $37^{\circ} \mathrm{C}$ in $5 \% \mathrm{CO}_{2}$. MTS assay was performed by transferring the discs to another TCPS plate, washing with PBS and incubating with $1 \mathrm{~mL}$ MTS mixture according to the procedure described above. OD was measured at $490 \mathrm{~nm}$ and percentage of cell viability was calculated by normalization with the mean OD value obtained for the TCPS culture. All tests were performed in triplicate $(n=9)$ (SilvaCorreia et al., 2012).

\subsubsection{NP cells encapsulation}

Prior to encapsulation within the GG-based hydrogel formulations, isolated NP cells were expanded until passage 4 (P4). After cell detachment from the culture flasks by $0.25 \%$ trypsin/EDTA solution, a diluted cell suspension was prepared and centrifuged at $1200 \mathrm{rpm}$ for $5 \mathrm{~min}$. For encapsulation of NP cells in the different formulations, the medium was completely aspirated and the cell pellet was re-suspended in the following hydrogel formulations: 1) $0.75 \%(\mathrm{w} / \mathrm{v})$ HA-GG; 2) 2\% (w/v) GG-GMA; 3) 25\%:75\% (v/v) HA-GG/GG-MA; 4) 50\%:50\% (v/v) HA-GG/GG-MA; and 5) 75\%:25\% (v/v) HA-GG/GG-MA. Cells and hydrogel were centrifuged at $600 \mathrm{rpm}$ for $3 \mathrm{~min}$ to allow homogenization of cells. The mixture was then used to produce 6mm diameter discs using a silicone mould (200 $\mu$ l per disc). The discs with the encapsulated NP cells (50 000 cells/disc) were then immersed in PBS ( $\mathrm{pH}$ 7.4) for at least $30 \mathrm{~min}$. Next, discs were incubated for periods of $1,3,7,14$ and 21 days in DMEM:F12 (1:1) medium at $37^{\circ} \mathrm{C}$ and $5 \% \mathrm{CO}_{2}$. Discs without cells (controls) were produced and incubated under similar conditions.

\subsubsection{Calcein-AM staining}

Cell viability after 1, 3, 7, 14 and 21 days was assessed using Calcein-AM staining. Briefly, a 1/1000 Calcein-AM solution (Molecular Probes, Eugene, OR, USA) was prepared in serum-free DMEM:F12 (1:1) medium without Phenol Red. The GG-based discs with the encapsulated rabbit NP cells were collected from the TCPS plates and incubated in $1 \mathrm{~mL}$ Calcein-AM solution for $15-30 \mathrm{~min}$ at $37^{\circ} \mathrm{C}$ and $5 \% \mathrm{CO}_{2}$ and then washed in sterile PBS. Samples were then observed under fluorescence microscopy using an Axio Imager Z1 light microscope with an attached AxioCam MRm digital camera connected to AxioVision image processing software (all from Carl Zeiss, Oberkochen. Germany).

\subsubsection{ATP quantification assay}

ATP quantification assay was used to determine the number of viable cells in cultures based on quantitation of ATP present, which signals the presence of metabolically active cells. At 1, 3, 7 and 14 days, the discs were transferred to another TCPS plate and washed three times with sterile PBS. Next, discs were incubated for $10 \mathrm{~min}$ in a 1:1 mixture of serum-free culture medium without Phenol Red and an ATP reagent (CellTiter-Glo ${ }^{\circledR}$ Luminescent Cell Viability Assay; Promega, USA) at room temperature. The mixture was transferred in duplicate to a 96-well black plate specific for luminescencebased assays and luminescence (sensitivity $=140$ ) was measured using a plate reader. ATP standards were prepared for construction of the calibration curve by consecutive dilutions of the ATP standard solution (provided by the kit) in PBS. Dilutions were also incubated with ATP reagent at a ratio of 1:1 and transferred to a 96-well black plate for luminescence measurement. Results are presented in relation to ATP concentration, which was directly proportional to cell number.

\subsection{Statistical analyses}

Paired student's t-test was used to determine statistical significance with significance set at $p<0.05,0.005$ and 0.001 .

\section{Results}

\subsection{Characterization of GG-based hydrogel formulations}

\subsubsection{DMA}

Different formulations of GG-based hydrogels were examined by DMA analysis. DMA results are presented in Figure 1, with (a) and (b) showing the storage modulus (E') and loss factor ( $\tan \delta$ ), respectively. Formulations composed by 0.75\% (w/v) HA-GG and 25\%:75\% (w/v) HA-GG:GG-MA were not sufficiently stable for DMA analysis. The storage modulus of $2 \%(\mathrm{w} / \mathrm{v})$ GG-MA was greater than all other formulations tested, even though it also behaved as a linear 




(a)

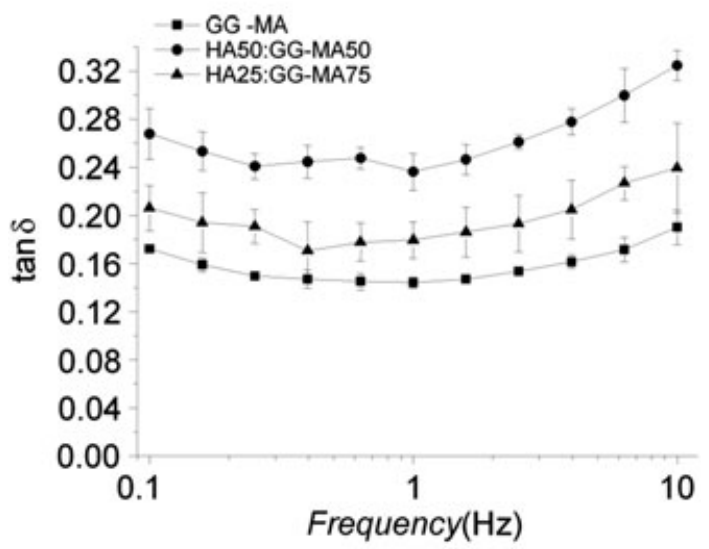

(b)

Figure 1. DMA analysis of different formulations of HAGG and ionic-crosslinked GG-MA hydrogel discs showing: (a) the storage modulus (E') and (b) loss factor $\left(\tan \delta\right.$ ) measured in PBS at $37^{\circ} \mathrm{C}$

viscoelastic material. Conversely, loss factor values for GG-MA were lower compared to other formulations.

\subsubsection{Degradation and swelling ability}

Weight loss and water uptake ability of HA-GG and GG-MA were determined by immersion in PBS solution for $180 \mathrm{~h}$ (Figure 2). Figure 2a shows the results of the degradation assay. In vitro degradation of GG formulations was investigated by measuring the weight loss rate of the matrix. Figure $2 \mathrm{~b}$ represents the swelling capacity of the two formulations following immersion in PBS for $170 \mathrm{~h}$. HA-GG showed significantly greater water uptake ability compared to GG-MA.

\subsection{In vitro cytotoxicity screening of $2 \mathrm{D}$ GG hydrogels}

\subsubsection{Morphology of isolated NP cells from rabbit IVDs}

Figure 3 showed morphology of NP cells at third passage after isolated from rabbit IVDs. NP cell maintained fibroblast-like shape during experimental periods without any changes of cell morphology.

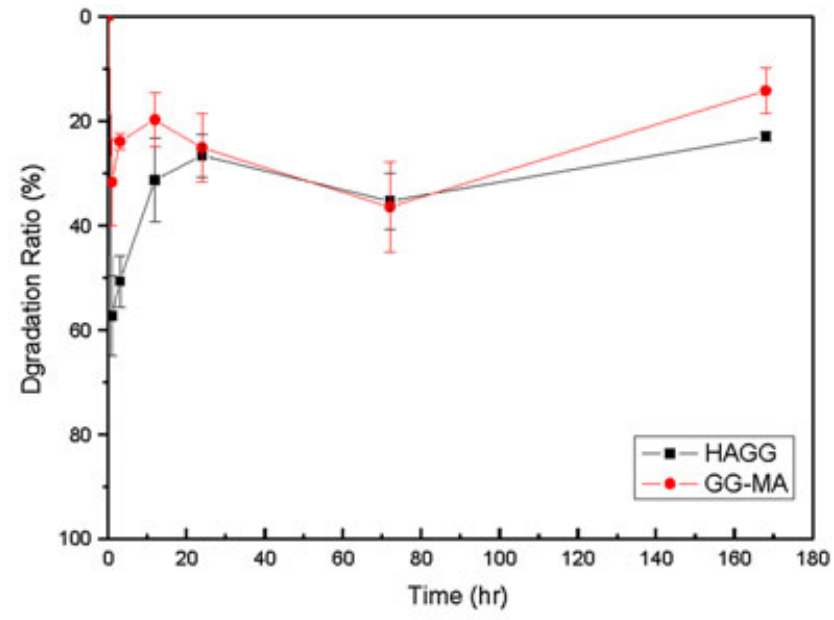

(a)

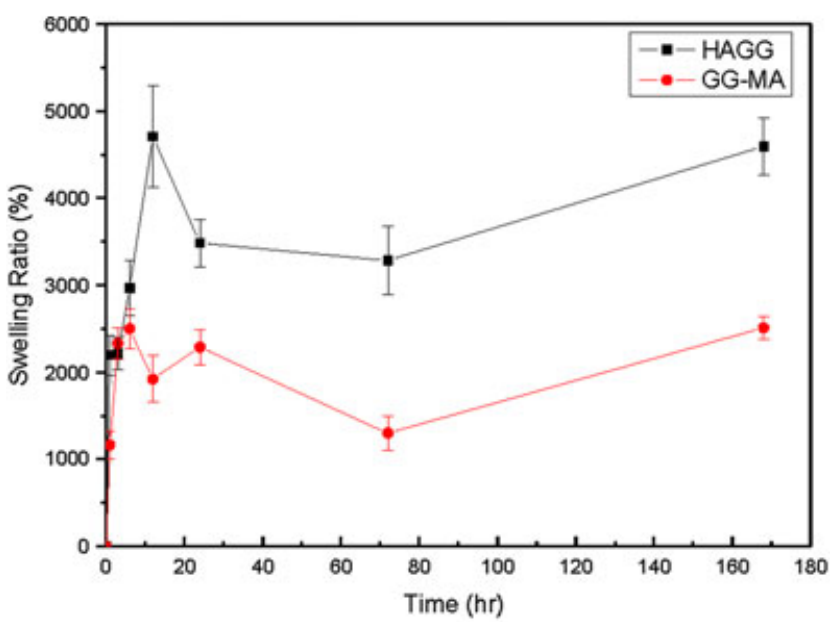

(b)

Figure 2. Degradation (a) and swelling kinetics (b) of HAGG and ionic-crosslinked GG-MA hydrogels. All samples were soaked in


180 hours

\subsubsection{In vitro cytotoxicity screening}

MTS assays were performed at 1, 3 and 7 days to evaluate NP cell viability and proliferation ability on GG-based hydrogels (Figure 4). MTS assays were also used to assess cytotoxicity in vitro. NP cells were used to screen for possible cytotoxicity. In particular, the high-acyl GG group maintained a greater number of viable cells compared to other hydrogels (Figure 5).

\subsection{In vitro biological evaluation of $3 \mathrm{D}$ GG-based hydrogels}

\subsubsection{NP cell encapsulation}

Different hydrogels were prepared by combining GG-MA and HA-GG in the following ratios: 25\%:75\% (w/v) HA-GG:GGMA; 50\%:50 \% (w/v) HA-GG:GG-MA; and 75\%:25\% (w/v) HA-GG:GG-MA. NP cells were cultured within the hydrogels for 1, 3, 7 and 14 days (Figure 6). 


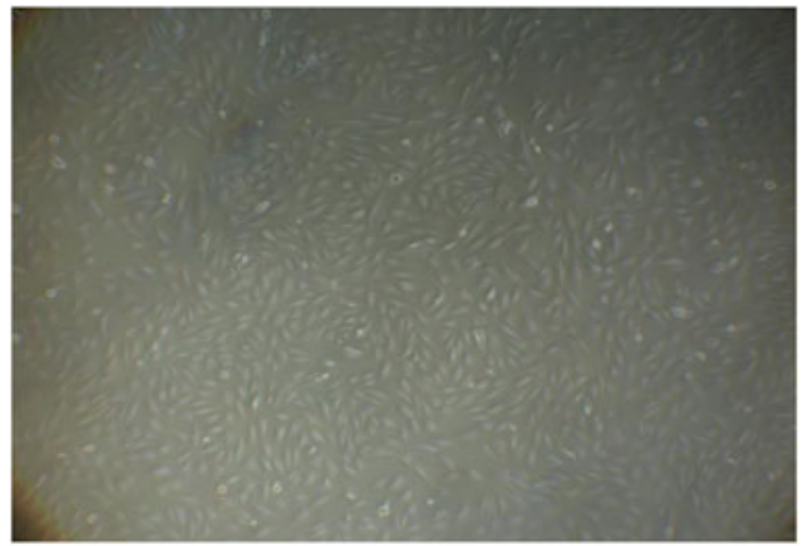

(a)



(b)

Figure 3. Morphology of nucleus pulposus (NP) cells in culture. Phase-contrast photomicrograph showing the cultured NP cells after third passage (magnification: (a) $50 \mathrm{x}$, (b) $100 \mathrm{x}$ )

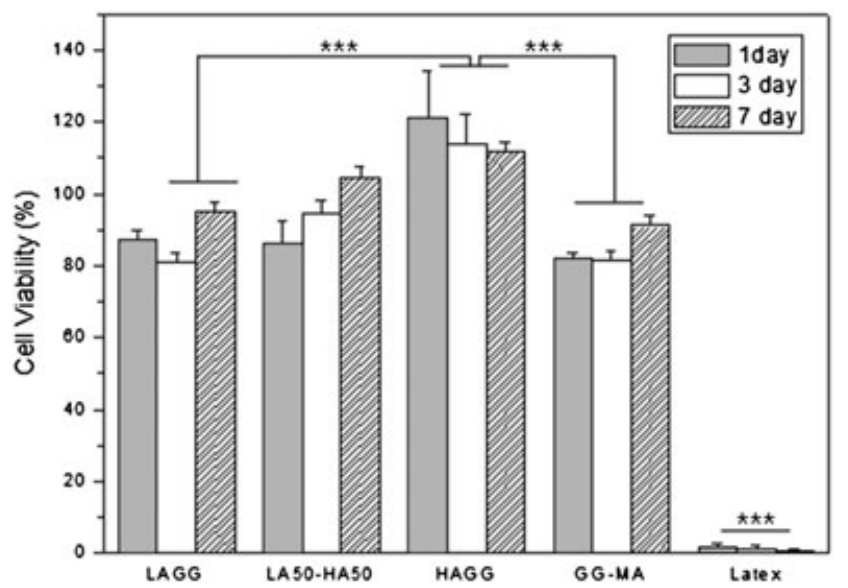

Figure 4. Cytotoxicity screening of the different formulations of gellan gum-based hydrogel leachables using mouse lung fibroblasts (L929 cells): high acyl gellan gum (HAGG) and ioniccrosslinked methacrylated gellan gum (GG-MA). Latex extract (Latex) and standard culture medium were used as positive and negative controls, respectively. Cell viability (\%) was measured following MTS assay after 1, 3 and 7 days in culture with the extract fluids. (* corresponds to $p<0.05$ and $* * *$ corresponds to $p<0.001$, in comparison with GG for each day)

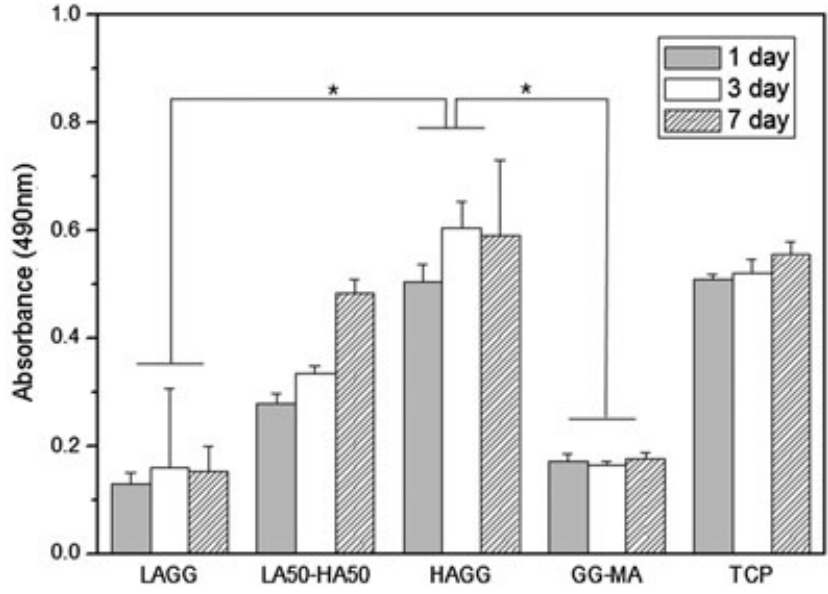

(a)



(b)

Figure 5. Measurement of cellular viability seeded NP onto the surface of gellan gum-based hydrogel. (a) The absorbance was measured after 1, 3 and 7 days. (b) Cell viability (\%).* Corresponds to $\mathrm{P}<0.05$ and ${ }^{* * *}$ corresponds to $\mathrm{P}<0.001$ in comparison with GG for each day

\subsubsection{Calcein-AM staining}

After encapsulation with NP cells, we performed a Calcein-AM assay to confirm cell viability at each period to determine the best conditions for cells to inhabit the hydrogel. Calcein-AM, which is strongly negativelycharged green fluorescent, has been widely used as a cell marker. This is because it is retained in the cytoplasm, which is then hydrolyzed by endogenous esterase. As a result, Calcein-AM easily confirmed cell viability since the cells that became extinct did not become green fluorescent. Calcein-AM assay was performed at 1 and 14 days. Results are presented in Figure 7. However, we did observe live cells that confirmed cell viability, since cells that became extinct could not became green fluorescent. All experiments in the current study included morphological assessments. NP cells had a round-shaped morphology following HA-GG and the shape did not change over time. In GG-MA, the shape of the cells was the same as HA-GG after encapsulation. 


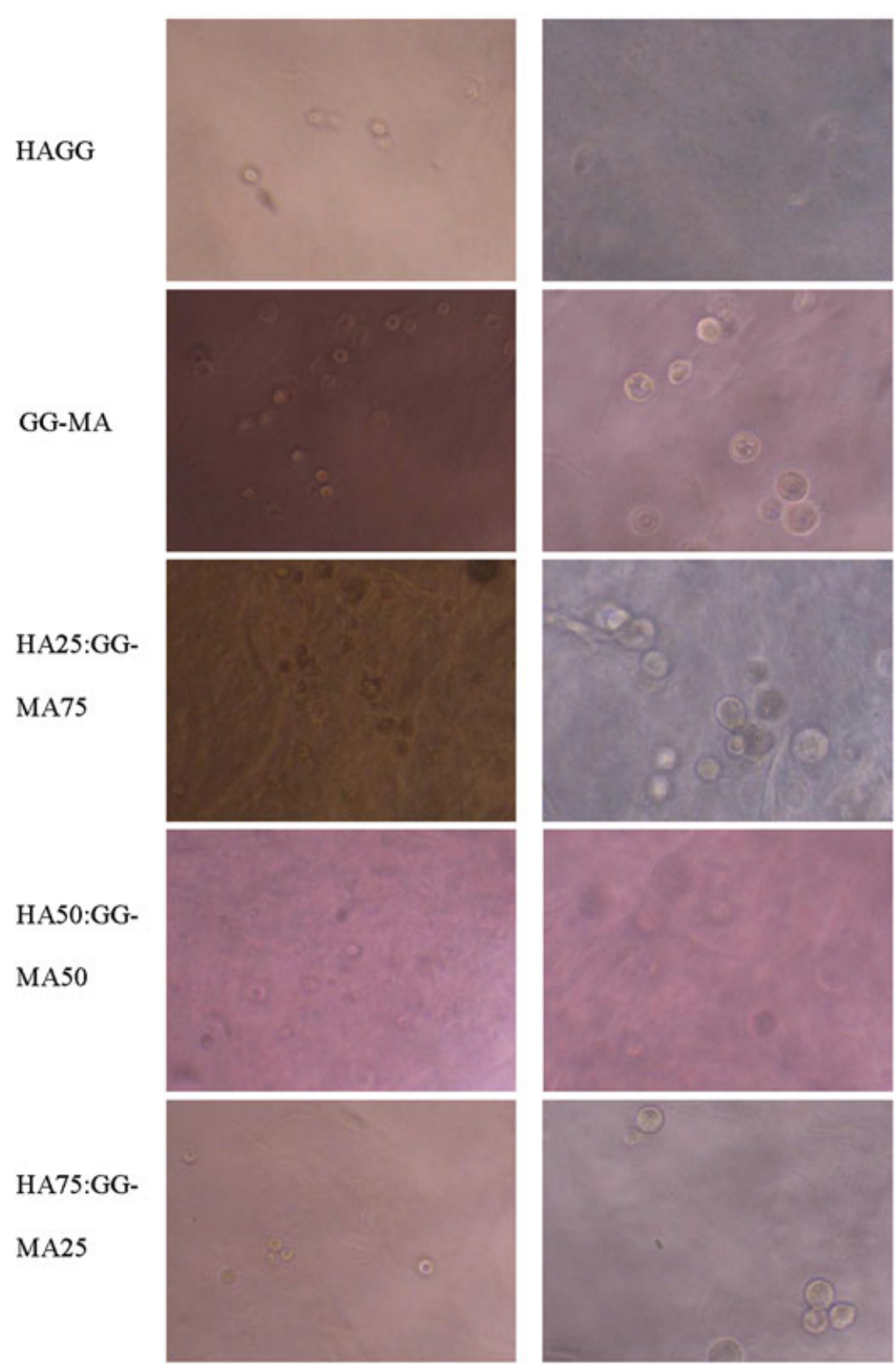

Figure 6. Microphotographs of gellan gum-based hydrogel encapsulated NP

\subsubsection{ATP quantification assay}

The ATP assay is highly sensitive for cell determination, proliferation and cytotoxicity based on luminescence signals that detect ATP activity. ATP concentrations vary due to cell growth and apoptosis. Cells maintain a limited concentration of ATP while alive. As a result, information about metabolic status and overall shape of cells can be obtained by measuring the intracellular concentration of ATP. Prior to ATP analysis, we tested whether the assays were suitable for GG hydrogels. Proliferation and cytotoxicity of encapsulated cells after culture are shown in Figure 8. Cells seeded on the scaffold gradually and significantly increased during all periods. From 1 to 14 days, NP cells in both GG-MA and 50\%:50\% (w/v) HAGG:GG-MA groups proliferated more than other groups. The GG-MA group showed slight decreases on day 14 while the 50\%:50\% (w/v) HAGG:GG-MA group increased steadily.

\section{Discussion}

Tissue regeneration is dependent on microenvironmental conditions and specific functions of the tissue being examined (Liu and Czernuszka, 2007). As a result, the mechanical and physical properties of the biomaterial used as assessed by DMA and swelling/degradation tests, respectively, are important in the development of scaffolds suitable for cell accommodation (Nemir \& West, 2009). Our results 


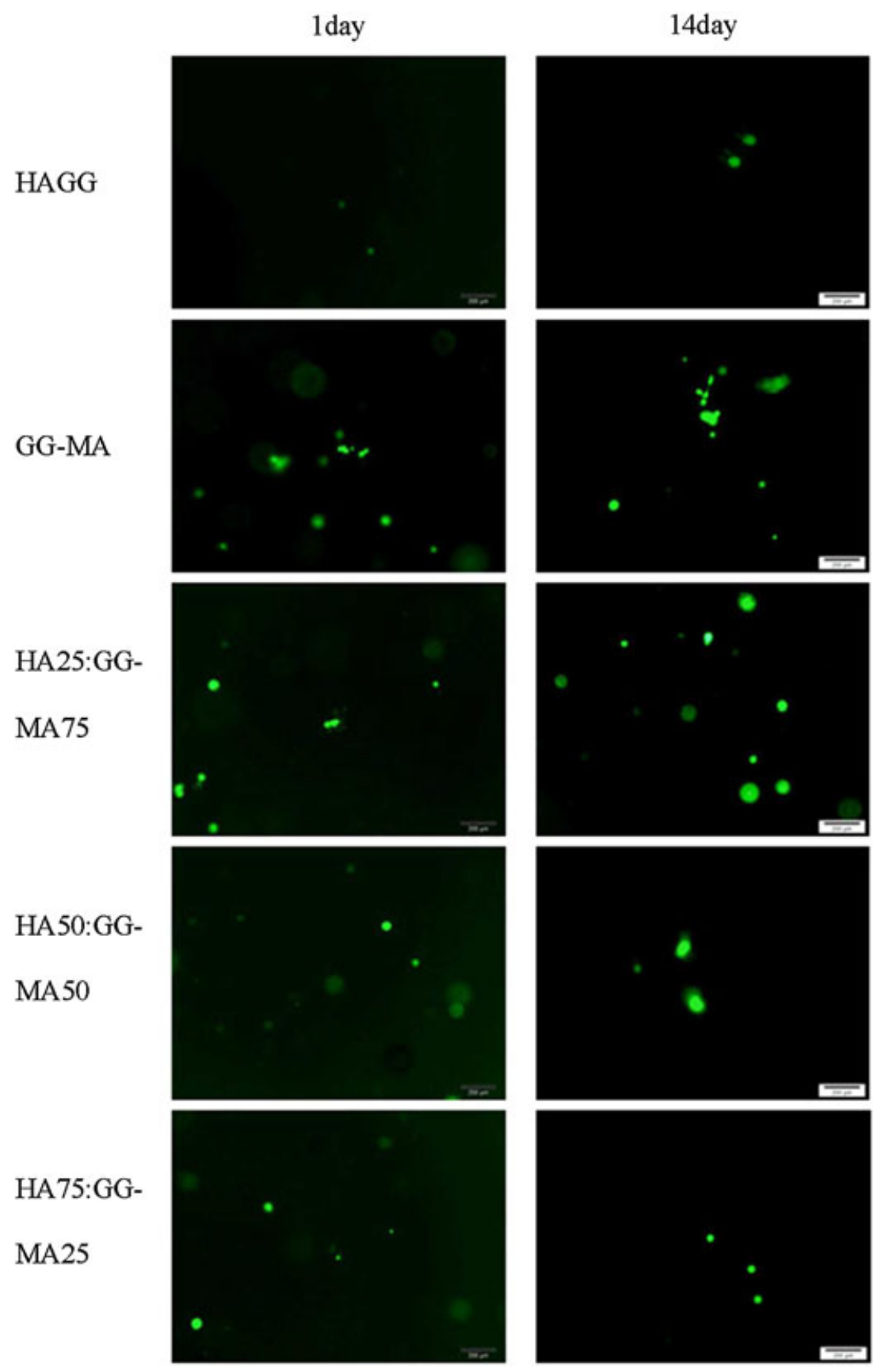

Figure 7. Representative fluorescence microphotographs of live encapsulated NP in gellan gum-based hydrogel cultured 1, 3, 7, 14 and 21 (magnification x100)

showed, as predicted, that a greater content in HA-GG resulted in reduced mechanical properties of the formulation. These findings, which are in accordance to results described by Silva-Correia et al. (2011), showed that GG-MA hydrogels were somewhat elastic and that the GG-MA formulation resulted in a more compact microstructure and stable condition in PBS. GG hydrogels are quite stable in PBS, especially when compared to other hydrogels (Miyoshi E et al., 1996). Compared to the other GG formulations examined in the current study, GG-MA proved to be the most stable.

Generally, hydrolytic degradation of polysaccharides such as GG hydrogel can be achieved in vivo through the activity of specific enzymes (Xia W et al., 2004). The effects of degradation ratios can be also result in cellular interaction and proliferation (Lee SK et al., 2010; Murphy WL et al., 2000). Therefore, it is importance to determine the degradation profile of biomaterials since too great a degradation rate can have an adverse impact on cell proliferation and viability (Oliveira JT et al., 2010). For this reason and according to the results obtained in DMA analyses, degradation of HA-GG was significantly faster compared to GG-MA hydrogels. These results are consistent with the fact that GG-MA hydrogels have a higher crosslinking density, and thus are more difficult to degrade. The swelling ability of HA-GG was greater than that of GG-MA hydrogels, which was in accordance with previous results reported by Silva-Correia J et al. (2011) that showed that the ionic-crosslinked methacrylated material was less able 


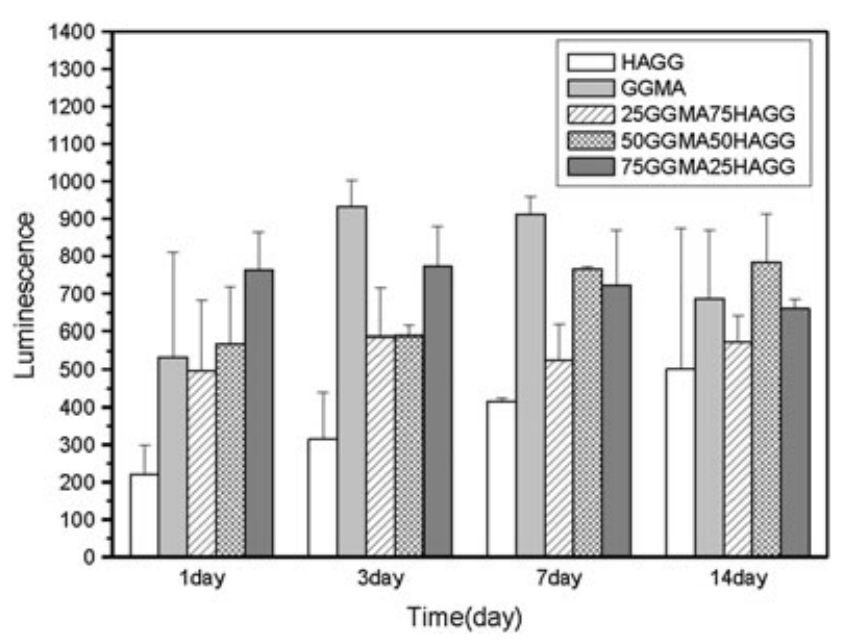

Figure 8. Measurement of cellular viability in gellan gum-based hydrogel encapsulated NP by ATP assay on cultivation after 1, 3,7 and 14

to swell due to its tighter matrix. According to previous studies, a greater swelling ability was shown to be strongly correlated with an accelerated hydrolysis reaction (Lee SK et al., 2010; Silva-Correia J et al., 2011). Characterization of the swelling ability of a biomaterial should be performed to optimize the exchange of metabolic products and nutrients. All formulations in the current study showed an elevated swelling ratio with values $>2000 \%$ after $3 \mathrm{~h}$ of immersion. Over time, GG-MA maintained its swelling ratio of $\sim 2000 \%$, whereas HA-GG was $>4000 \%$. These results demonstrate that HA-GG incorporation into the GG hydrogels increased weight loss of the hydrogels.

Screening of cytotoxicity was performed in 2D cultures and 3D scaffolds using NP cells (Figure 4) to evaluate the effects of the different formulations on cell viability and growth. For 2D cultures, an MTS assay was performed to evaluate the effect of the biomaterials on cell metabolism and viability. This test was performed based on the assumption that if the material was toxic, that cells would react by inhibiting growth, decreasing metabolism, by cell death or changes in cell morphology. Cytotoxicity results are shown in Figure 4. These data show that HA-GG had a significant influence on NP attachment of cells under 2D culture conditions. It was observed that the hydrogels did not have a deleterious effect on NP cell viability during all periods tested. It was also observed that NP cells attached to 96-well TCPS plates had a typical morphology (spindle- to squamous-shaped) similar to the morphology observed in the culture flasks, whereas cells cultured in latex extracts were round-shaped. In all formulations, cell viability was $\sim 100 \%$ of control, thereby confirming that the materials were non-cytotoxic. The $0.75 \%(\mathrm{w} / \mathrm{v})$ HA-GG formulation allowed the greatest percentage of cell viability ( $>100 \%$ ), demonstrating that this composition offered the best conditions for NP cell growth compared to others. As a result, it can confidently stated that the different formulations of GG-based hydrogels tested were safe for NP cells in terms of cytotoxicity and also bio-affinity. In addition, biological evaluations of the formulations were also performed by seeding NP cells on 3D hydrogel discs. 2D in vitro culturing showed that GG-based hydrogels provided an adequate environment for adhesion and proliferation of NP cells. In addition, we chose only two of the three materials, which provided greater attachment and proliferation of NP cells.

To study the viability of NP cells in 3D culture, NP cells were encapsulated within the different GG hydrogels. All hydrogels were found to support normal functioning of NP cells. We also observed that NP cells were distributed homogeneously in the hydrogels. The typical shape of NP cells was squamous-shaped (Figure 3) but round-shaped cells were observed when encapsulated within the different hydrogels.

For applications in tissue engineering, it is important to show cell viability and proliferation under 3D conditions (Mauney et al., 2005). We demonstrated by Calcein-AM analysis that NP cells maintained their initial morphology. Conversely, NP cells adopted a spindle-shaped morphology in GG-MA depending on duration. Distribution and compaction of encapsulated cells were greater in high-acyl than other groups. These changes were easier to confirm over time. A greater ratio of GG-MA contributed more to the proliferation and viability of NP cells compared to examining each group according to their different GG hydrogel ratios. A favourable condition for biomaterial construction is superior interaction between cells and scaffolds (Robert, 2001). Our results therefore confirmed not only the biocompatibility of GG-MA for cell encapsulation but also that it accelerated metabolism of NP cells. The cultured disc encapsulated with GG was assessed by cytotoxicity measurements to identify proliferation and viability of NP cells in GG hydrogel. As a result, we assessed MTS by extraction tests and 2D for cytotoxicity but did not find any tendencies in the encapsulated disc. ATP assay was performed to quantitatively evaluate the viability of NP cells encapsulated within the different hydrogels. ATP levels corroborated Calcein-AM assay findings that revealed that as the ratio of GG-MA increased, a corresponding increase in cell viability and growth was observed. Results of cell viability and proliferation on 2D cultures and 3D scaffolds were different in the current study. Considering the differences between HA-GG that showed the most influence on $2 \mathrm{D}$ and GG-MA that showed the most influence on $3 \mathrm{D}$, the HA-GG group also showed increased luminescence during culture. Data showed continuous increases in cell viability and proliferation in HA-GG compared to GG-MA groups.

Based on previous studies (Gong YH et al., 2009; Oliveira JTb et al., 2009a; Oliveira JT et al., 2009b; Oliveira JT et al., 2010; Silva-Correia $\mathrm{J}$ et al., 2011), HA-GG is a superior biomaterial for cell proliferation and viability due to its similarity to natural extracellular matrix (Anderson DG et al., 2005). Nevertheless, cell proliferation on HA-GG occurred as a result of weakness of the mechanical properties; important factors in biomaterial. In addition, biomaterial should develop structurally to allow transport of nutrients and metabolites from the cells (Anderson DG et al., 2005; Vunjak-Novakovic G., 2003). The GG-MA group showed the greatest increase in luminescence compared to HA-GG over time. These data show that the GG-MA group 
was unable to support cell viability and proliferation. In addition, increases in the GG-MA ratio also increased luminescence. This implies that GG-MA is a more suitable material than HA-GG for biocompatibility under 3D conditions. Additionally, the 50\%:50\% (w/v) HAGG:GG-MA group maintained continuously greater cell viability and proliferation and was determined to be the best ratio for NP cells.

\section{Conclusions}

The study investigated the biological performance of a gellan gum-based hydrogel culture using rabbit NP cells. Different GG-based hydrogels were produced from methacrylated GG-MA and HA-GG. Results revealed that HA-GG decreased the mechanical properties and stability of GG-MA/HA-GG hydrogel formulations. However, the addition of HA-GG to the GG-MA hydrogels promoted the proliferation of NP cells when cultured onto the surface of the hydrogels. Cell culture experiments also revealed that all GG-based hydrogel formulations were non-cytotoxic and supported NP cell viability up to 21 days of culture. As a result, we demonstrated that methacrylated gellan gum and formulations possessing high-acyl gellan gum present tunable properties that could be interesting for applications as NP substitutes.

\section{Conflict of Interest}

The authors declare no conflict of interest.

\section{Acknowledgements}

The authors thank the Portuguese Foundation for Science and Technology (FCT) for financial support through POCTI and FEDER programmes. This work was also conducted with the support of the European Union funded Collaborative Project Disc Regeneration (NMP3-LA-2008-213904), MBC(A04003) and the World Class University project (R31-20029).

\section{References}

Anderson DG, Risbud MV, Shapiro IM et al. 2005; Cell-based therapy for disc repair. Spine 5: 297-303.

Bibby SRS, Jones DA, Lee RB et al. 2001; The pathophysiology of the intervertebral disc. Joint Bone Spine 68(6): 537-542.

Bogduk N. 1997; Clinical Anatomy of the Lumbar Spine and Sacrum 2nd Eds. 13.

Chung HJ, Park TG. 2009; Self-assembled and nanostructured hydrogels for drug delivery and tissue engineering. Nano Today 4(5): 429-437.

Coviello T, Dentini M, Rambone G et al. 1998; A novel co-crosslinked polysaccharide: studies for a controlled delivery matrix Original Research Article. $J$ Control Release 55(1): 57-66

Fritz JM, Cleland JA, Speckman M et al. 2008; Physical therapy for acute low back pain: associations with subsequent healthcare costs. Spine 33: 1800-1805.

Gong YH, Wang CM, Lai RC et al. 2009; An improved injectable polysaccharide hydrogel: modified Gellan gum for long-term cartilage regeneration in vitro. J Mater Chem 19(14): 1968-1977.

Hoffman AS. 2002; Hydrogels for biomedical applications. Adv Drug Deliv Rev 54(1): 3-12.

Hu SG, Jou CH, Yang MC. 2003; Protein adsorption, fibroblast activity and antibacterial properties of poly(3-hydroxybutyric acidco-3-hydroxyvaleric acid) grafted with chitosan and chitooligosaccharide after immobilized with hyaluronic acid. Biomaterials 24(16): 2685-2693.

ISO/EN10993-5. 2009; Biological evaluation of medical devices - Part 5: Tests for in vitro cytotoxicity. International Standards Organization: Genève, Switzerland.

Jansson PE, Lindberg B, Sandford PA. 1983; Structural studies of gellan gum, an extracellular polysaccharide elaborated by Pseudomonas elodea. Carbohydr Res 124(1): 135-139.

Khang G, Lee SJ, Kim MS et al. 2006; Biomaterials: Tissue-Engineering and Scaffolds. In Encyclopedia of Medical Devices and
Instrumentation (2nd Eds). John Wiley \& Sons, Inc: NJ, USA, 366-383.

Khang G, Lee SJ, Lee JM et al. 2002; Polymercell interaction. In Tissue Engineering and Regenerative Medicine, Koonja: Korea, Chap. 17: 297-322.

Lee KY, Mooney DJ. 2001; Hydrogels for tissue engineering. Chem Rev 101: 1869-1880.

Lee SK, Hong HK, Kim SJ et al. 2010; The comparison of sponges and PLGA scaffolds impregnated with DBP on growth behaviors of human intervertebral disc cells Polymer (Korea), 34(5): 398-404.

Liu CZ, Czernuszka JT. 2007; Development of biodegradable scaffolds for tissue engineering: a perspective on emerging technology. Mater Sci Tech 23(4): 379-391.

Manchikanti L, Singh V, Datta S et al. 2009; Comprehensive review of epidemiology, scope, and impact of spinal pain. Pain Physician 12: 35-70.

Matricardi P, Cencetti C, Ria R et al. 2009; Preparation and characterization of novel Gellan gum hydrogels suitable for modified drug release. Molecules 14(9): 3376-3391.

Mauney JR, Jaquiery C, Volloch V et al. 2005; In vitro and in vivo evaluation of differentially demineralized cancellous bone scaffolds combined with human bone marrow stromal cells for tissue engineering. Biomaterials 26: 5173-3185.

Miyoshi E, Takaya T, Nishinari K. 1996; Rheological and thermal studies of gelesol transition in Gellan gumaqueous solutions. Carbohydr Polym 30: 109-119.

Murphy WL, Peters MC, Kohn DH et al. 2000; Sustained release of vascular endothelial growth factor from mineralized poly(lactide-co-glycolide) scaffolds for tissue engineering. Biomaterials 21(24): 2521-2527.

Nakamura K, Harada K, Tanaka Y et al. 1993; Viscoelastic properties of aqueous gellan solutions: the effects of concentration on gelation. Food Hydrocolloids 7(5): 435-447.
Nanjawade BK, Manvi FV, Manjappa AS. 2007; In situ-forming hydrogels for sustained ophthalmic drug delivery. $J$ Control Release 122(2): 119-134.

Nemir S, West JL. 2009; Synthetic materials in the study of cell response to substrate rigidity. Ann Biomed Eng 38(1): 2-20.

Oliveira JT, Martins L, Picciochi R et al. 2009a; Gellan gum: a new biomaterial for cartilage tissue engineering applications. J Biomed Mater Res A 93A: 852-863.

Oliveira JT, Santos TC, Martins L et al. $2009 \mathrm{~b}$; Performance of new gellan gum hydrogels combined with human articular chondrocytes for cartilage regeneration when subcutaneously implanted in nude mice. J Tissue Eng Regen Med 3: 493-500.

Oliveira JT, Santos TC, Martins L et al. 2010; Gellan gum injectable hydrogels for cartilage tissue engineering applications: in vitro studies and preliminary in vivo evaluation. Tissue Eng Part A 16: 343-353.

O'Neill MA, Selvendran RR, Morris VJ et al. 1983; Structure of the acidic extracellulargelling polysaccharide produced by Pseudomonas elodea. Carbohydr Res 124(1): 123-133.

Pereira DR, Silva-Correia J, Oliveira JM et al. 2011a; Hydrogels in acellular and cellular strategies for intervertebral disc regeneration. J Tissue Eng Regen Med wileyonlinelibrary.com, DOI: 10.1002/term.500

Pereira DR, Silva-Correia J, Caridade SG et al. 2011b; Development of Gellan gum-based microparticles/hydrogel matrices for application in the intervertebral disc regeneration. Tissue Eng Part C 17(10):961-972.

Robert L. 2001; Matrix biology: past, present and future. Pathol Biol 49: 279-283.

Sanderson GR. 1990; Food gels. Elsevier Applied Science: New York; 201-202.

Silva-Correia J, Oliveira JM, Oliveira JT et al. 2010; Photo-crosslinked Gellan gum-based hydrogels: methods and uses thereof. WO2011/119059, Priority date: 105030 26.03.2010 PT. 


\section{Biological evaluation of gellan gum-based hydrogels}

Silva-Correia J, Oliveira JM, Caridade SG et al. 2011; Gellan gum-based hydrogels for intervertebral disc tissue-engineering applications. J Tissue Eng Regen Med 5: e97-e107.

Silva-Correia J, Zavan B, Vindigni V et al. 2012; Biocompatibility evaluation of ionicand photo-crosslinked methacrylated gellan gum hydrogels: in vitro and in vivo study. Adv Healthcare Mater. DOI: 10.1002/adhm.201200256

Vunjak-Novakovic G. 2003; The fundamentals of tissue engineering: scaffolds and bioreactors. Novartis Found Symp 249(34-46): 170-174.

Weiner AA, Moore MC, Walker AH et al. 2008; Modulation of protein release from photocrosslinked networks by gelatin microparticles. Int $J$ Pharm 360(1-2): 107-114.

Xia W, Liu W, Chi L et al. 2004; Tissue engineering of cartilage with the use of chitosan-gelatin complex scaffolds. $J$ Bio Mater Res App 71B(2): 373-380. 